\title{
Leukocyte Localization in a Model of Innate Immune-Driven Colitis
}

\author{
Jordan R. Jones ${ }^{1}$, Anne-Marie C. Overstreet ${ }^{2}$, Antonia M. Boger-May ${ }^{2}$, David L. \\ Boone $e^{2,3}$ \\ ${ }^{1}$ Indiana University School of Medicine, ${ }^{2}$ Indiana University School of Medicine, \\ Department of Microbiology and Immunology, ${ }^{3}$ University of Notre Dame
}

\begin{abstract}
Background and Hypothesis:
Inflammatory bowel disease (IBD) is a disabling, chronic gut disorder involving immune dysregulation. Our lab has generated a murine IBD model in which the innate immune system drives inflammation. Innate lymphoid cells (ILCs), an innate immune cell subset that was recently discovered, exhibit many T-helper cell characteristics. ILCs, though few, produce cytokines, thereby significantly impacting tissue through local action in mucosal sites. They express the cell surface markers, CD90, which is unique to ILCs, and CD45, which all leukocyte types express. Colitis prevention in our model via ILC depletion indicates a role for ILCs in IBD. Therefore, we aimed to identify the ILCs' localization in our murine model. We hypothesized that the ILCs will localize to inflamed areas of the intestinal lamina propria and into the intraepithelial spaces.
\end{abstract}

\section{Experimental Design or Project Methods:}

Mice expressing TNFAIP3, an inhibitor of NF-kB, were mated with adaptive immunity-lacking mice (RAG $1^{-/}$). RAG $1^{-/-}$x Villin-TNFAIP3 (TRAG) mice had colitis that was $100 \%$ penetrant by age 6 weeks. Distal colons excised at age 4 weeks and 8 weeks were used for identifying $\mathrm{CD} 45^{+}$and $\mathrm{CD} 90^{+}$cells in both RAG and TRAG mice intestines via immunofluorescence.

\section{Results:}

We observed differences in the distributions of $\mathrm{CD}^{+} 0^{+}$and $\mathrm{CD} 45^{+}$cells within TRAG and RAG mice intestines.

\section{Conclusion and Potential Impact:}

Differences exist in intestinal leukocyte distributions within our models. Altered ILC distribution might reflect an inflammatory state or contribute to IBD pathology. This work may further elucidate ILCs' role in IBD and as IBD treatment targets. 